\title{
Fast 1D Inversion of Logging-While-Drilling Resistivity Measurements for Improved Estimation of Formation Resistivity in High-Angle and Horizontal Wells
}

\author{
David Pardo* and Carlos Torres-Verdín ${ }^{\dagger}$ \\ *Department of Applied Mathematics, Statistics, and Operational Research, \\ University of the Basque Country (UPV/EHU), Leioa, Spain, \\ Basque Center for Applied Mathematics (BCAM), and IKERBASQUE (Basque \\ Foundation for Sciences), Bilbao, Spain. \\ ${ }^{\dagger}$ The University of Texas at Austin, Austin TX, USA.
}

(December 14, 2014)

Running head: Inversion of LWD Resistivity Measurements

\begin{abstract}
We introduce an efficient inversion method to estimate layer-by-layer electrical resistivity from logging-while-drilling (LWD) electromagnetic induction measurements. The method assumes a one-dimensional (1D) model based on planarly layered transversely isotropic (TI) formations with known bed boundaries, penetrated by arbitrary well trajectories. Forward simulations are based on a 1D reduced model where both borehole and mandrel effects are assumed negligible. The stopping criteria, regularization term, regularization parameter, Jacobian matrix, and inversion variables are automatically estimated by the inversion algorithm, thereby minimizing the required user interaction and expertise. Numerical inversion results of challenging synthetic and field data confirm the high stability and superior approximation properties of the developed inversion algorithm. Results indicate that tri-axial
\end{abstract}


induction measurements provide significantly more stable inversion results than conventional co-axial measurements, especially in the presence of anisotropic formations. 


\section{INTRODUCTION}

Interpretation of resistivity measurements acquired in high-angle and horizontal wells remains one of the most critical problems in formation evaluation. Presence of thin beds, anisotropy, and variable borehole trajectory can give rise to abnormal raw and apparent resistivity logs that cannot be interpreted without corrections. Apparent resistivity "horns" and abnormal separation of multi-resolution resistivity curves are two common diagnostic situations of interpretation problems. Without numerical simulation, it becomes impossible to detect, quantify, and reduce those effects to reliably assess hydrocarbon saturation. It is necessary to invoke the most efficient simulation methods and the most robust inversion procedures to produce practical, efficient, and reliable automatic inferences of true layer resistivities and dielectric permittivities devoid of shoulder bed, geometric, and other environmental effects.

A number of three-dimensional (3D) numerical simulators of borehole resistivity measurements have been developed during the last two decades for oil-industry applications (e.g., Zhang et al. (1995); Druskin et al. (1999); Newman and Alumbaugh (2002); Davydycheva et al. (2003); Wang and Fang (2001); Wang and Signorelli (2004); Avdeev et al. (2002); Pardo et al. (2007, 2008b,a); Nam et al. (2008, 2010)). These simulators have been successfully used to study and quantify different physical effects occurring in 3D geometries. Despite such recent advances, there are still many 3D effects for which reliable inversion methods are not available. The lack of $3 \mathrm{D}$ inversion results (as opposed to $2 \mathrm{D}$ results) is due to major difficulties encountered when solving geometrically challenging problems. Specifically, for mesh-based methods such as finite elements, finite differences, and boundary elements, the size of the system of linear equations becomes excessively large to be solved in 
real (logging) time, preventing their efficient inversion in the context of logging-while-drilling (LWD) devices.

Thus, many service and oil companies base some of their inversion processes on a reduced model composed of planar transversely isotropic (TI) layered media with unknown piecewise constant resistivities. For this problem, semi-analytical solutions can be easily computed in a fraction of a second (see Kong (1972); Chew and Chen (2003), and more recently, Løseth and Ursin (2007); Zhong et al. (2008) and Streich and Becken (2011)), enabling the fast simulation of borehole resistivity measurements. The idea of using a reduced model for inversion of resistivity LWD measurements was already proposed over 20 years ago by Meyer (1993); Merchant et al. (1996), and Anderson (2001), among others. These initial results were further developed in Ijasan et al. (2014), where the bed boundaries were determined first from nuclear measurements, followed by an estimation of electrical properties within each layer based on resistivity measurements. This latter work only employed data acquired with one specific LWD resistivity device, and no tri-axial induction instruments were considered in the interpretation.

In our work, we also assume a planarly layered TI formation with known bed boundaries that have been pre-determined, either from nuclear logging measurements or from some other possibly available information. In either case, we assume that the given bed boundary positions are correct, and we restrict the study only to those situations. Forward simulations are based on a 1D reduced model where both borehole and mandrel effects are assumed negligible, as illustrated in Figure 1. Given one or various apparent resistivity logs, a description of the logging instrument, the well trajectory, and the bed boundaries, the inversion method developed here delivers: (a) a layer-by-layer resistivity distribution that minimizes the misfit between the measurements and their numerical simulations, and (b) 
the corresponding uncertainty bars expressing the degree of uncertainty of inversion results. Because the reference system of coordinates can be properly rotated, we assume without any loss of generality that bed boundaries are horizontal.

[Figure 1 about here.]

The main contribution of our work is the development and description of an inversion method of resistivity measurements under the above assumptions. It works with any commercial logging device with known antennae configurations, including tri-axial instruments. The inversion method requires no user expertise, as it automatically determines the weights, regularization parameter, Jacobian matrix, inversion variable, and stopping criteria. Because of the efficiency, flexibility, and stability of the inversion algorithm, it can be readily employed by formation-evaluation specialists for routine analysis and appraisal of complex LWD and wireline resistivity measurements acquired under general geometrical and geological constraints.

We specify all pertinent details about the mathematical formulation of our inversion method, and study its application under noisy measurement conditions, as well as the robustness of inversion results under different borehole and/or formation conditions, including anisotropy, and variable dip angles and logging instruments. Of particular interest is the comparison of inverted results corresponding to conventional LWD resistivity tools with multiple antennas and operating frequencies against those obtained with tri-axial induction measurements and a single pair of antennas operating at a single frequency. We also examine the inversion of field data.

In commercial logging instruments, both multiplicative and additive noise may be present, being the additive noise more noticeable at low frequencies. Due to that condition, spe- 
cific tool-dependent weights are needed to diminish the importance of low-frequency noisy measurements during inversion. To avoid such analysis, here we restrict the study to only multiplicative Gaussian noise in the acquired measurements. We only observe minor effects of such type of noise in the inversion results.

The remainder of this paper is organized as follows: First, we focus on the simulation of the forward problem using a semi-analytical solution, with especial emphasis on the computation of apparent resistivities. Second, we describe our inversion method. A few details on the implementation and computational complexity are described next. Experimental results using both synthetic examples as well as field measurements illustrate the salient features of our inversion algorithm, including its limitations. Finally, we emphasize the most preeminent conclusions concerning best practices for implementation.

\section{FORWARD METHOD}

\section{D Semi-Analytical Solution}

In the second part of the last century, several researchers developed a semi-analytical solution for the electromagnetic field radiated by a magnetic dipole in planarly layered media, both for isotropic (Wait (1951)) and TI (Wait (1966); Kong (1972)) formations. Under the assumption that formation properties are constant in the horizontal direction (see Figure 1b), semi-analytical solutions are derived by following these steps:

1. A Hankel transform in the horizontal plane. This is equivalent to using two Fourier transforms, reducing the original partial differential wave equation into an ordinary differential equation (ODE) that depends upon the vertical direction. 
2. The resulting ODE is solved analytically in terms of transmission and reflection coefficients that enforce proper interface conditions among consecutive layers.

3. The final solution is computed by numerically evaluating the inverse Hankel transform of the above spectral solution.

The semi-analytical solution is expressed in terms of the corresponding magnetic field components in the Cartesian system of coordinates, namely,

$$
\mathbf{H}_{C}=\left(\begin{array}{ccc}
H_{C}^{x x} & H_{C}^{x y} & H_{C}^{x z} \\
H_{C}^{y x} & H_{C}^{y y} & H_{C}^{y z} \\
H_{C}^{z x} & H_{C}^{z y} & H_{C}^{z z}
\end{array}\right),
$$

where the first super-index designates the dipole direction of the source, and the second one specifies the measured field component, both expressed in the Cartesian system of coordinates.

\section{Apparent Resistivities}

In order to compare the above semi-analytical results to LWD resistivity measurements, we need to first transform the former into the system of coordinates of the logging instrument. For that purpose, we realize that the Cartesian system of coordinates $\left(x_{C}, y_{C}, z_{c}\right)$ can be obtained by rotating the borehole system of coordinates $\left(x_{B}, y_{B}, z_{B}\right)$ first with respect to the dip angle $\theta$ and then with respect the azimuthal angle $\phi$. Mathematically, following the 
axis orientation depicted in Figure 2 (Zhong et al. (2008)), we have:

$$
\left(\begin{array}{c}
x_{C} \\
y_{C} \\
z_{C}
\end{array}\right)=\underbrace{\left(\begin{array}{ccc}
\cos \phi & -\sin \phi & 0 \\
\sin \phi & \cos \phi & 0 \\
0 & 0 & 1
\end{array}\right)}_{R_{z}} \cdot \underbrace{\left(\begin{array}{ccc}
\cos \theta & 0 & \sin \theta \\
0 & 1 & 0 \\
-\sin \theta & 0 & \cos \theta
\end{array}\right)}_{R_{y}} \cdot\left(\begin{array}{c}
x_{B} \\
y_{B} \\
z_{B}
\end{array}\right) .
$$

Thus, the magnetic field in the borehole system of coordinates $\mathbf{H}_{B}$ is computed as:

$$
\mathbf{H}_{B}=R^{-1} \mathbf{H}_{C} R
$$

where $R=R_{z} \cdot R_{y}$, and $R^{-1}=R^{T}$ due to the properties of rotations.

[Figure 2 about here.]

For the case of tri-axial induction tools, in this work we consider all components of the magnetic field $\mathbf{H}_{B}$ without post-processing, which requires to know the magnetic moments of the transmitters and receivers. Most commercial tools provide some type of (post-processed) apparent resistivities which may have lost some amount of information contained in the original magnetic field results.

In conventional LWD instruments, the above results are further post-processed to obtain apparent resistivities. The main ideas used to perform this operation are delineated in the works of Bonner et al. (1995) and Clark et al. (1988). First, we compute the $z_{B}$-component of the magnetic field excited by a $z_{B}$-oriented dipole at two different receivers $R X_{1}$, and $R X_{2}$. We denote them by $H_{B}^{z z}\left(R X_{1}\right)$ and $H_{B}^{z z}\left(R X_{2}\right)$, respectively. Second, we compute the logarithm of its ratio, which can be expressed using basic properties of complex numbers as

$$
\log \frac{H_{B}^{z z}\left(R X_{1}\right)}{H_{B}^{z z}\left(R X_{2}\right)}=\underbrace{\log \left(\frac{\left|H_{B}^{z z}\left(R X_{1}\right)\right|}{\left|H_{B}^{z z}\left(R X_{2}\right)\right|}\right)}_{\text {Attenuation }}+i \underbrace{\left\{p h\left(H_{B}^{z z}\left(R X_{1}\right)\right)-p h\left(H_{B}^{z z}\left(R X_{2}\right)\right)\right\}}_{\text {Phase difference }}
$$


where $p h$ indicates the phase of a complex number. Third, we compute numerically the relation between the above numbers (attenuation and phase difference) with the resistivity of a homogeneous formation, as illustrated in Figure 3. Finally, this transformation is recorded for each combination of one transmitter and two receivers, and applied to all measurements (including those acquired in heterogeneous formations), thereby transforming magnetic fields into apparent resistivities. Notice that some tool manufacturers include in this transformation the effect of an artificial permittivity function that is often (although not always) assumed to be a function of formation resistivity (see Zhou (2012)). In this work, we assume that LWD measurements are already environmentally corrected, including corrections for borehole and dielectric effects.

[Figure 3 about here.]

There is a common belief within the logging industry that when a formation is symmetric with respect to a plane perpendicular to the borehole, the recorded LWD measurements should also exhibit the same type of symmetry. To achieve this effect, most tools incorporate a set of transmitters whose locations are symmetric with respect to the center of the two receivers. However, some manufacturers minimize the number of transmitters to reduce costs. Subsequently, they perform a "numerical symmetrization" that consists of computing a linear combination of the measured results at the existing transmitters/receivers in such a way that they recover the aforementioned "symmetry condition" in most borehole settings. Unfortunately, the actual coefficients of this linear combination are often confidential. Due to this situation, here we avoid such numerical symmetrization, and for non-symmetrical tools we simply add the necessary transmitters in order for the logging instrument to become symmetric. 


\section{INVERSION METHOD}

\section{Inversion Problem.}

We start by defining the following cost functional:

$$
C_{W}(s)=\|H(s)-M\|_{l_{W_{M}}^{2}}^{2}+\lambda\left\|s-s_{0}\right\|_{L_{W_{s_{0}}}^{2}}^{2}
$$

where

- $l_{W_{M}}^{2}$ and $L_{W_{s_{0}}}^{2}$ denote the discrete $\left(l_{2}\right)$ and continuous $\left(L_{2}\right)$ weighted norms with weights $\left(W_{M}\right)_{i}=\frac{1}{\left\|M_{i}\right\|_{l^{2}}}$ and $\left(W_{s_{0}}\right)_{i i}=\frac{1}{\left\|s_{0, i}\right\|_{L^{2}}}$, respectively,

- $s=s(\rho)$ is a variable that depends upon the resistivity $\rho$, for example, $s=\log \rho$, $s=\frac{1}{\rho}$, or $s=\rho$,

- $M$ is the set of field (or synthetic) noisy measurements expressed in terms of tri-axial induction measurements or apparent resistivities. The method also allows one to perform the inversion with arbitrary magnetic field components (for general tri-axial induction tools) or with any other post-processed quantity.

- $H(s)$ is the set of simulated measurement for a given $s$,

- $\lambda$ is a regularization (or stabilization) parameter that adjusts the relative weight given to the two additive components of the above cost functional to obtain stable results, and

- $s_{0}$ is an a priori distribution of $s$.

We assume that the resistivity distribution $\rho$ is constant in the horizontal direction and piecewise constant in the vertical direction $z$ with known bed-boundaries, i.e., that it can 
be expressed as $\rho=\rho(z)=\sum_{i} \rho_{i} \chi_{i}(z)$, where $\chi_{i}(z)$ is equal to 1 within the $i$-th layer, and 0 elsewhere. Then, given a set of (possibly noisy) measurements $M$, the objective of the inversion algorithm is to estimate the resistivity distribution coefficients $\rho_{i}^{*}$ such that $\rho^{*}:=\arg \min _{\rho} C_{W}(s(\rho))$.

\section{Inversion Algorithm.}

To solve the above minimization problem, we select the following deterministic iterative scheme:

$$
s\left(\rho^{(n+1)}\right)=s\left(\rho^{(n)}\right)+\delta s\left(\rho^{(n)}\right)
$$

where superscript $n$ designates the iteration number, and $\delta s\left(\rho^{(n)}\right)$ is the $n$-th increment to the solution. To simplify the notation, we define $s^{(n)}:=s\left(\rho^{(n)}\right)$ and $\delta s^{(n)}:=\delta s\left(\rho^{(n)}\right)$.

For conventional LWD tools, we select as initial resistivity distribution $\rho^{(0)}$ the average of the measured apparent resistivities within each layer. For tri-axial tools, we select $\rho^{(0)}=1$.

To determine $\delta s^{(n)}$, we first take a Taylor's series expansion of $H\left(s^{(n+1)}\right)$ in the neighborhood of $s^{(n)}$ of the form:

$$
H\left(s^{(n+1)}\right) \approx H\left(s^{(n)}\right)+\underbrace{\left(\frac{\partial H\left(s^{(n)}\right)}{\partial s}\right)}_{J_{s}^{(n)}} \delta s^{(n)},
$$

Then, by solving equation $\frac{\partial C_{W}\left(s^{(n+1)}\right)}{\partial \delta s^{(n)}}=0$, we obtain the Gauss-Newton's method:

$$
\delta s^{(n)}:=-\frac{\operatorname{Re}\left(J_{s}, H\left(s^{(n)}\right)-M\right)_{l_{W_{M}}^{2}}+\lambda\left(I, s^{(n)}-s_{0}\right)_{L_{W_{s_{0}}}^{2}}}{\operatorname{Re}\left(J_{s}^{(n)}, J_{s}^{(n)}\right)_{l_{W_{M}}^{2}}+\lambda(I, I)_{L_{W_{s_{0}}}^{2}}}
$$




\section{Jacobian Matrix}

To compute the Jacobian matrix $J_{s}^{(n)}$, we first employ the chain rule to obtain

$$
J_{s}^{(n)}=\frac{\partial H\left(s^{(n)}\right)}{\partial s}=\underbrace{\frac{\partial H\left(s^{(n)}\right)}{\partial \rho}}_{J_{\rho}^{(n)}} \frac{\partial \rho}{\partial s} .
$$

The second factor on the right-hand side $\frac{\partial \rho}{\partial s}$ is computed analytically, while the first term is numerically approximated using the definition of derivative, namely,

$$
J_{\rho}^{(n)}=\frac{\partial H\left(s^{(n)}\right)}{\partial \rho} \approx \frac{H\left(s\left(\rho^{(n)}+h\right)\right)-H\left(s\left(\rho^{(n)}\right)\right)}{h}
$$

where $h$ is a small number (perturbation size). Notice that $h$ should be at the same time small enough to properly approximate the above derivative and sufficiently large to avoid magnification of numerical errors arising from the forward solver. Thus, a proper choice of $h$ depends upon the particular forward solver employed to perform the simulations. In our case, we select $h$ such that the relative difference between the simulated measurements in a homogeneous formation with background resistivity $\rho$ and $\rho+h$ is approximately $1 \%$. This parameter can be further adjusted as a function of the error of the inverse problem. We emphasize that $h$ depends upon the distance between transmitter and receivers, frequency of operation, and background resistivity.

It is also possible to compute the Jacobian matrix using the semi-analytical formulas that appear in Streich and Becken (2011). This latter approach delivers accurate solutions without the need to select the perturbation size $h$. However, it requires a significantly more complex implementation.

$J_{\rho}^{(n)}$ is a matrix with as many columns as the number of measurements and as many rows as the number of layers (basis functions for $\rho^{(n)}$ in the formation). The $i j$-th entry of 
matrix $J_{\rho}^{(n)}$ is expressed as:

$$
\left\{J_{\rho}^{(n)}\right\}_{i j} \approx \frac{H_{i}\left(s\left(\rho^{(n)}+h_{j}\right)\right)-H_{i}\left(s\left(\rho^{(n)}\right)\right)}{h_{j}} .
$$

From the above formula, we conclude that computing the Jacobian matrix requires $N_{L A Y E R S}+$ 1 evaluations of the solution per logging position and iteration. On the other hand, once $J_{\rho}^{(n)}$ is found, we can calculate $J_{s}^{(n)}$ at a negligible cost using Equation (8).

In this work, we have selected six different free variables, $s$, namely: (a) $s=\rho$, (b) $s=\sigma$, (c) $s=\log _{10} \rho$, (d) $s=\log _{e} \rho$ (e) $s=\rho^{2}$, and (f) $s=\sqrt{\rho}$. Within each iteration, we compute the entries of the Jacobian matrix for all six variables, evaluate the error, and select the one that delivers the largest error reduction. We have experimentally observed that different variables are selected at each iteration of the problem, leading to improved robustness and faster error reduction than using any single variable $s$.

\section{Regularization Parameter.}

The regularization term is intended to avoid ill-posedness of the problem, hence it should be sufficiently large. At the same time, we would like this term to be as low as possible so that the cost functional is mainly driven by the measurement misfit. As a compromise between the above two contradictory conditions, several algorithms can be employed to adjust the regularization parameter. One of the most popular methods for selecting the regularization parameter is the so-called L-curve algorithm Hansen and O'Leary (1993); Hansen (1999), which is invoked in this paper. Notice that the regularization parameter $\lambda=\lambda^{(n)}$ becomes iteration dependent.

Another alternative studied in this paper consists of selecting the regularization term to account for a given percentage of the total cost functional, for example, $10 \%$. An exact 
computation of the regularization term to achieve the above goal is prohibitively expensive from the computational point of view. Rather, we employ the Taylor's series expansion of Equation (7) to approximate:

$$
C_{W}\left(s_{\lambda^{(n)}}^{(n+1)}\right) \approx\left\|H\left(s^{(n)}\right)+J_{s}^{(n)} \delta s_{\lambda^{(n)}}^{(n)}-M\right\|_{l_{W_{M}}^{2}}^{2}+\lambda^{(n)}\left\|s^{(n)}+\delta s_{\lambda^{(n)}}^{(n)}-s_{0}\right\|_{L_{W_{s_{0}}}^{2}}^{2},
$$

Then, we select

$$
\lambda^{(n)}:=0.1 * \frac{\left\|H\left(s^{(n)}\right)+J \delta s_{\lambda(n)}^{(n)}-M\right\|_{l_{W_{M}}^{2}}^{2}}{\left\|s^{(n)}+\delta s_{\lambda^{(n)}}^{(n)}-s_{0}\right\|_{L_{W_{s_{0}}}^{2}}^{2}} .
$$

A fixed-point iteration is performed to obtain the value of $\lambda^{(n)}$. Using this algorithm, we arrive at significantly different regularization parameters than those obtained using the Lcurve algorithm; nonetheless, the quality of inverted results in the examples examined below remains comparable.

\section{Stopping Criteria.}

We stop the inversion when both the relative data misfit and regularization terms become almost invariant as a function of the iteration number. Consistent with the idea that the regularization term only contributes with $10 \%$ of the total cost functional, and requesting that the total variation of the cost functional from iteration $n$ to iteration $n+1$ be below $1 \%$, we impose the following two stopping conditions:

$$
\begin{gathered}
\frac{\left|\left\|H\left(s^{(n+1)}\right)-M\right\|_{l_{W_{M}}^{2}}^{2}-\left\|H\left(s^{(n)}\right)-M\right\|_{l_{W_{M}}^{2}}^{2}\right|}{\|M\|_{l_{W_{M}}^{2}}^{2}} \leq \frac{0.5}{100}(=0.5 \%) \\
\lambda^{(n)} * \frac{\left|\left\|s^{(n+1)}-s_{0}\right\|_{L_{W_{s_{0}}}^{2}}^{2}-\left\|s^{(n)}-s_{0}\right\|_{L_{W_{s_{0}}}^{2}}^{2}\right|}{\left\|s_{0}\right\|_{L_{W_{0}}^{2}}^{2}} \leq \frac{5}{100}(=5 \%) .
\end{gathered}
$$

We stop the inversion algorithm when both conditions are satisfied or the maximum number of iterations is reached. 


\section{Measurements, Inversion Parameters, and a Priori Resistivity Distribu- tion}

LWD devices acquire multiple measurements per logging position. For the inversion, the developed algorithm allows one to consider only a subset of the measurements in order to: (a) speed up computations, and (b) minimize possible noise effects occurring at certain frequencies and spacings between transmitters and receivers. Similarly, it is also possible to reduce the number of inversion parameters to minimize the ill-posedness of the problem, for example, by selecting an isotropic model rather than a transversely isotropic (TI) one.

The algorithm also allows one to increase the number of measurements and/or inversion parameters in subsequent iterations of the inversion process, although the specific selection of measurements and inversion parameters in each iteration is decided by the user.

The a priori data $s_{0}=s\left(\rho_{0}\right)$ is also selected by the user. In the absence of additional information coming from surface and/or other logging measurements or geological information, one selects $s_{0}:=s^{(0)}$.

\section{Uncertainty Bars.}

Along with the final solution, it is desirable for an inversion algorithm to provide an error analysis intended to appraise the stability and uniqueness of the inverse solution due to noise effects and/or the selected assumptions that may not hold in practice. Ideally, the error analysis should produce a set of solutions for which the cost functional only varies slightly. Computing this range of solutions in actual logging situations is nearly impossible. Rather, a set of computable uncertainty bars is often delivered in replacement of the aforementioned error study. 
As indicated by their name, uncertainty bars deliver a tensor-product subset of solutions for which the cost functional only varies slightly. Their computation requires the solution of multiple inverse problems with multiple initial data, and their cost is several times that of solving the inverse problem. Thus, their computational cost is prohibitively expensive in our application. To minimize this cost, we assume that after achieving convergence, $\delta s^{(n)} \approx 0$. By considering new noisy measurements of the type $\widetilde{M}=M+N$ and using these new measurements in the Gauss-Newton method, we obtain the following new correction after the first iteration:

$$
\widetilde{\delta s^{(n)}}(N):=\frac{\left.\operatorname{Re}\left(J_{s}^{(n)}, N\right)\right)_{l_{W_{M}}^{2}}}{\operatorname{Re}\left(J_{s}^{(n)}, J_{s}^{(n)}\right)_{l_{W_{M}}^{2}}+\lambda^{(n)}(I, I)_{L_{W_{s_{0}}}^{2}}}
$$

The cost of computing $\widetilde{\delta s^{(n)}}(N)$ is negligible, and various corrections may be computed for different noise realizations. In our method, for a given type of noise (for example, a $10 \%$ normal Gaussian distribution), we compute 100 noise vectors $N_{i}$ and the corresponding $\widetilde{\delta s^{(n)}}\left(N_{i}\right)$ and define the (linearized) uncertainty bar as:

$$
\left[s^{(n)}+\min _{i} \widetilde{\delta s^{(n)}}\left(N_{i}\right), s^{(n)}+\max _{i} \widetilde{\delta s^{(n)}}\left(N_{i}\right)\right]
$$

The aforementioned uncertainty bars can be described as linearized uncertainty bars in the neighborhood of the attained local minimum, and they should be cautiously interpreted to avoid misleading interpretations. In particular, they provide no information of other possibly existing minima and/or nonlinear behavior of the cost function. While a large uncertainty bar does indicate large uncertainty, a small uncertainty bar is no guarantee of an accurate result. Interested readers are referred to Fernández-Martínez et al. (2013) for additional details about uncertainty quantification. 


\section{IMPLEMENTATION DETAILS AND COMPUTATIONAL COMPLEXITY.}

While the existing literature (e.g., Chew and Chen (2003); Løseth and Ursin (2007); Streich and Becken (2011)) reflects the wide use of semi-analytical solutions for geophysical exploration, the question of how to efficiently implement them is barely examined. Once a proper quadrature rule is selected, even a mediocre implementation provides an accurate solution for a single transmitter-receiver combination, frequency, and logging position in a fraction of a second.

Unfortunately, during the inversion of LWD resistivity measurements, the number of forward problems that need to be solved may quickly grow beyond $10^{6}$, leading to a prohibitively expensive method. To examine this situation, we first analyze the amount of time required to solve an inverse LWD problem using the above semi-analytical solution. A single evaluation of the solution requires the following amount of time $T(E)$ (evaluation time):

$$
T(E) \approx C * N_{I N T} * N_{L A Y E R S},
$$

where $C$ is a constant, $N_{I N T}$ is the number of integration points, and $N_{L A Y E R S}$ is the number of layers. A full forward simulation requires the solution to be evaluated at all logging positions and for all transmitters, receivers, and operating frequencies. Therefore, the time employed by a full forward simulation $T(F)$ is given by:

$$
T(F) \approx N_{P O S} * N_{T X} * N_{R X} * N_{F R E Q} * T(E)
$$

where $N_{P O S}$ is the number of logging positions, $N_{T X}$ is the number of transmitters, $N_{R X}$ is the number of receivers, and $N_{F R E Q}$ is the number of frequencies. Finally, the solution of the inverse problem involves the computation of the entries of the Jacobian matrix. Thus, 
the total cost of the inversion process can be expressed as

$$
T(I) \approx N_{I T} * \underbrace{N_{L A Y E R S} * T(F)}_{\text {COST OF COMPUTING THE JACOBIAN }}
$$

where $N_{I T}$ is the number of iterations of the inverse problem. Consequently, even a solution evaluation time as low as $T(E)=0.1 s$ is prohibitively expensive in real borehole situations, whereby the use of highly efficient implementations is needed.

A possible path to improve the computational efficiency comes from the use of proper integration rules in combination with a fast inverse Hankel transform. For that purpose, we employ the algorithm developed in Anderson (1982), which requires below $N_{I N T}=100$ integration points in most borehole situations, although other alternative methods may also be invoked (e.g., Key (2012)).

A second common strategy that is widely employed to gain computational efficiency is to minimize the number of repeated operations that occur when executing multiple simulations. For example, it is typical to compute only one set of transmission and reflection coefficients for all receivers. The objective is to eliminate the dependence of $T(F)$ upon $N_{R X}$. A drawback of this approach is that one needs to select the same integration points for all receivers, which may increase $N_{I N T}$ and/or reduce the accuracy; it also increases the complexity of the implementation.

To circumvent the above problems and secure additional computational savings, we follow a different approach. First, we divide the well trajectory into sections that share a fixed dip and azimuthal angles of incidence, as depicted in Figure 1b. Within each section, we observe that the horizontal distance between each transmitter-receiver combination is constant for all logging positions. Therefore, integration points which depend upon the horizontal distance between transmitter and receiver may be fixed, and transmission and 
reflection coefficients become the same for all logging positions. Thus, we only compute these coefficients once, thereby reducing the overall cost of the algorithm by a factor equal to $\min \left\{N_{L A Y E R S}, \tilde{N}_{P O S}\right\}$, where $\tilde{N}_{P O S}$ is the number of logging positions with fixed azimuth

and dip. For most well trajectories, $\min \left\{N_{L A Y E R S}, \tilde{N}_{P O S}\right\}=N_{L A Y E R S}$. In this case, the overall cost of our inversion algorithm becomes

$$
T(I) \approx N_{I T} * N_{L A Y E R S} * N_{P O S} * N_{T X} * N_{R X} * N_{F R E Q} * N_{I N T} * C .
$$

Additionally, we have implemented a windowing system to enforce that when a particular layer is far away (typically, from 3 to $5 \mathrm{~m}$ ) from the tool position, such layer is removed from the computations, thus effectively reducing the number of layers $N_{L A Y E R S}$ per logging position.

\section{NUMERICAL RESULTS}

For the numerical results examined in this section, we assume the tool configurations described in Table 1, which are also shown in Zhou (2012). The first three correspond to commercial LWD devices with known transmitter-receiver configurations which only acquire the component of the magnetic field along the borehole direction, while the fourth one is a simple tri-axial induction instrument that measures all components of the magnetic field. Logging measurements are acquired every half a foot along the well trajectory, except for the synthetic case with thin layers, where the density of logging points is fourfold.

[Table 1 about here.] 


\section{Synthetic Examples}

We first examine a synthetic example containing layers of various thicknesses. The thickness of the thinnest and thickest layers are $0.38 \mathrm{~m}$, and $1.52 \mathrm{~m}$, respectively, while the resistivity of the formation ranges from $1.26 \Omega \cdot \mathrm{m}$ to $54.77 \Omega \cdot \mathrm{m}$, and the dip angle is constant throughout the well trajectory and equal to 82 degrees (unless otherwise specified). This example is intended to appraise the effectiveness of the inversion algorithm in a highly deviated well with realistic formations, including layers of various thicknesses and resistivity contrasts.

Figure 4 displays the results from the inversion for various logging instruments. In all cases we observe a good estimation of the actual layer resistivities, although some discrepancies exist. Such discrepancies originate mainly with the thinnest layers, whose resistivity is more difficult to estimate. In the thickest layers, inverted results agree well with the actual layer resistivities. The best results are obtained when employing a tri-axial induction tool, as it can be concluded from the error evolution displayed in Figure 5. These results suggest that a single frequency of operation and only one spacing between transmitter and receivers in a tri-axial induction tool provides more valuable (independent) information than three frequencies and five different spacings between transmitter and receivers in a conventional LWD tool.

[Figure 4 about here.]

[Figure 5 about here.]

Figure 6 displays the results obtained from the inversion for several dip angles using Tool 3. A good agreement is observed between actual and estimated resistivities for all cases. 
[Figure 6 about here.]

Figure 7 describes the inversion results obtained for a transversely isotropic (TI) formation and various dip angles. For the case of a conventional LWD instrument (top panel), we are able to accurately estimate only the horizontal resistivity for vertical wells, and the vertical resistivity for highly-deviated wells, as tool physics indicates. For example, in the layer located at $0.7<z<1.6 \mathrm{~m}$, the vertical resistivity is severely overestimated in a vertical well, since co-axial measurements obtained in a vertical well are indeed insensitive to the vertical resistivity. Horizontal conductivity estimations are very accurate throughout the entire well trajectory. For the deviated wells, we observe a much better estimation of both vertical and horizontal resistivities (except for the thinnest layers, whose resistivities are more difficult to estimate). When employing a tri-axial induction tool (bottom panel), we accurately estimate both vertical and horizontal resistivities, regardless of dip angle.

[Figure 7 about here.]

We now consider an example of thinly bedded formation, where the thickness of the thinnest and thickest layers are $0.05 \mathrm{~m}$ and $0.21 \mathrm{~m}$, respectively. This example is much more challenging than the previous one from the numerical point of view. Figure 8 shows the corresponding inversion results obtained for various logging instruments. Again, we observe that the acquisition of tri-axial induction measurements gives rise to significantly more accurate results. For this example, the inversion algorithm fails to estimate the exact resistivities using measurements acquired with Tool 1 in the thinnest layers, indicating that additional measurements are needed to properly estimate the resistivity of thinly bedded formations. 
[Figure 8 about here.]

Figures 9 and 10 display inversion results obtained for our original synthetic example as well as the example of thinly bedded formation in the presence of multiplicative Gaussian noise. Measurements are numerically simulated with Tool 3. While the assumed noise may generate some discrepancies on inversion results with respect to noise-free measurements, the inversion algorithm remains robust, even for the case of a thinly bedded formation. Disparities between actual and estimated resistivities increase due to the effect of noise, especially, for the case of thinly bedded formations. The largest differences between estimated and actual resistivities occur for the case of a $10 \%$ noise. As physically expected, uncertainty bars corresponding to the thinly bedded formation are significantly longer than those associated with the original synthetic example. Despite this relatively high uncertainty level, we emphasize that the developed inversion method estimates layer resistivities far more accurately than with apparent resistivities, leading to an improved description of the formation, and ultimately to an improved estimation of water saturation. In particular, we estimate formation resistivities of over $50 \Omega \cdot \mathrm{m}$ in layers where apparent resistivities do not exceed $15 \Omega \cdot \mathrm{m}$ at any logging point.

[Figure 9 about here.]

[Figure 10 about here.]

\section{Inversion of Field Measurements}

We now consider field measurements acquired with a conventional LWD instrument, specifically, with Tool 2. In the first example (Figure 11), there is a highly deviated well with a 
trajectory that advances over $150 \mathrm{~m}$ in the horizontal direction while only $4.2 \mathrm{~m}$ in the vertical direction. Additionally, the dip angle is above 90 degrees in some segment of the well trajectory. Bed boundaries have been previously determined with nuclear measurements. Notice that resistivity variations for this field example are relatively small (from $0.3 \Omega \cdot \mathrm{m}$ to $6 \Omega \cdot \mathrm{m})$.

[Figure 11 about here.]

In the second field example (Figure 12), the well penetrates a thinly bedded formation. For this well-log section, the well trajectory exhibits a constant dip angle of $79.31^{\circ}$. Again, resistivity variations for this thinly bedded example are limited (from $0.2 \Omega \cdot \mathrm{m}$ to $4 \Omega \cdot \mathrm{m}$ ).

[Figure 12 about here.]

Figure 13 displays the relative data misfits (data residuals) in percentage for the two considered field examples. In both cases, we observe a considerable data misfit reduction from our initial guess (average apparent resistivity within each layer) to the final inversion results. Despite the achieved improvement, we still observe some large data misfits in certain areas of the formation such as, for example, in field A at horizontal positions between 1030 $\mathrm{m}$ and $1040 \mathrm{~m}$. These data misfits may be due to the use of inaccurate bed boundary positions or 3D effects, and they suggest that the model should be updated on those areas.

[Figure 13 about here.]

We now analyze the computer efficiency of the inversion method. In terms of the required memory, only a few Mb are needed to invert even the most complex well and model 
conditions. In terms of CPU time, this is highly dependent upon the particular example under consideration. To illustrate this situation and show that the scalability predicted by Equation (20) is indeed achieved, we display in Table 2 the amount of time per iteration as well as the time per unit, which corresponds to the average time required to perform a single forward simulation (in milliseconds) for a particular receiver, transmitter, frequency, and logging position. Numerical results confirm that this is indeed a constant, as predicted. Of course, some variations occur (up to a factor of 2 or 2.5) because the predicted scalability is only achieved at the asymptotic level, while in the pre-asymptotic level some erratic behavior may take place.

From the practical point of view, for the two field examples considered in this paper, the CPU time needed to invert the acquired measurements ranged from half an hour to approximately 3 hours, depending upon the number of required iterations. Notice that in this paper we have not invoked a parallelization scheme, which may easily increase the speed of computations by a factor of ten when using only 10-15 processors.

[Table 2 about here.]

\section{CONCLUSIONS}

We developed a method for the fast 1D inversion of LWD resistivity measurements; it assumes a planarly layered 1D formation, and it is well-suited for highly deviated wells. Inversion results confirm the robustness, efficiency, and stability of the method for any dip angle, presence of noise, different tool configurations, and TI anisotropy. We also verified the inversion method with field measurements.

The numerical calculation of the entries of the Jacobian matrix is one of the most rele- 
vant components of the developed inversion method; its computation may be lengthy even when employing semi-analytical methods. To overcome this problem, we accelerated significantly the forward simulator by pre-computing common material data that are employed at various logging positions. Additionally, the perturbation size, $h$, used to numerically calculate the entries of the Jacobian matrix, should be properly adjusted according to each particular frequency and transmitter-receiver spacing in order to control numerical errors and attain convergence. Another critical part of the developed method is the regularization (stabilization) parameter, which should be properly adjusted to remove noise and ill-conditioning. Experience with the inversion algorithm applied to synthetic and field examples indicates that the latter two parameters are crucial for the reliable estimation of layer-by-layer resistivities from LWD measurements in real time.

Results indicate that the inversion method cannot overcome physical limitations imposed by conventional LWD resistivity measurements, for instance, when attempting to estimate vertical resistivities in vertical wells. Tri-axial/directional induction measurements provide substantially richer information to estimate layer properties than traditional LWD measurements based on sampling only the $H_{z z}$ (borehole co-axial) magnetic field component. Numerical results also confirm that the error and uncertainty levels of inversion results across thinly bedded formations are much larger than those associated with thick layers, as physically expected. Across thinly bedded formations, we accurately estimate resistivities of over $50 \Omega \cdot \mathrm{m}$ in well segments where the largest apparent resistivity does not exceed 15 $\Omega \cdot \mathrm{m}$, emphasizing the need and superiority of this type of inversion method over direct interpretation of apparent resistivities.

Future work will consider the parallelization of the inversion algorithm to target small parallel machines, to enable the calculation of inversion results in real time. For that 
purpose, each frequency, transmitter-receiver combination, and column of the Jacobian matrix will be executed in a different core/processor. We shall also analyze the influence of additive noise and/or incorrect bed boundary positions on the inverted results.

\section{ACKNOWLEDGMENT}

The work reported in this paper was funded by University of Texas at Austin's Research Consortium on Formation Evaluation, jointly sponsored by Afren, Anadarko, Apache, Aramco, Baker-Hughes, BG, BHP Billiton, BP, Chevron, China Oilfield Services, LTD., ConocoPhillips, ENI, ExxonMobil, Halliburton, Hess, Maersk, Marathon Oil Corporation, Mexican Institute for Petroleum, Nexen, ONGC, OXY, Petrobras, PTT Exploration and Production, Repsol, RWE, Schlumberger, Shell, Statoil, TOTAL, Weatherford, Wintershall and Woodside Petroleum Limited.

The first author was partially funded by the RISE Horizon 2020 European Project GEAGAM (644602), the Project of the Spanish Ministry of Economy and Competitiveness with reference MTM2013-40824-P, the BCAM "Severo Ochoa" accreditation of excellence SEV-2013-0323, the CYTED 2011 project 712RT0449, and the Basque Government through the BERC 2014-2017 program and the Consolidated Research Group Grant IT649-13 on "Mathematical Modeling, Simulation, and Industrial Applications ( $\left.\mathrm{M}^{2} \mathrm{SI}\right)$ ".

We are grateful to Dr. Olabode Ijasan for his insightful discussions on the topic. 


\section{REFERENCES}

Anderson, B. I., 2001, Modeling and Inversion Methods for the Interpretation of Resistivity Logging Tool Response: PhD thesis, Delft University of Technology.

Anderson, W. L., 1982, Fast hankel transforms using related and lagged convolutions: ACM Transactions on Mathematical Software (TOMS), 8, no. 4, 344-368.

Avdeev, D. B., A. V. Kuvshinov, O. V. Pankratov, and G. A. Newman, 2002, Threedimensional induction logging problems, part 1: An integral equation solution and model comparisons: Geophysics, 67, 413-426.

Bonner, S., J. Tabanou, P. Wu, J. Seydoux, K. Moriarty, B. Seal, E. Kwok, and M. Kuchenbecker, 1995, New 2-mhz multiarray borehole-compensated resistivity tool developed for mwd in slim holes: Society of petroleum engineers.

Chew, W., and S. Chen, 2003, Response of a point source embedded in a layered medium: Antennas and Wireless Propagation Letters, IEEE, 2, no. 1, 254-258.

Clark, B., M. Luling, J. Jundt, M. Ross, and D. Best, 1988, A dual depth resistivity measurement for fewd: Presented at the SPWLA 29th Annual Logging Symposium,(Jun. 1988).

Davydycheva, S., V. Druskin, and T. Habashy, 2003, An efficient finite-difference scheme for electromagnetic logging in 3D anisotropic inhomogeneous media: Geophysics, 68, no. $5,1525-1536$.

Druskin, V. L., L. A. Knizhnerman, and P. Lee, 1999, New spectral Lanczos decomposition method for induction modeling in arbitrary 3-D geometry: Geophysics, 64, no. 3, 701706.

Fernández-Martínez, J. L., Z. Fernández-Muñiz, J. Pallero, and L. M. Pedruelo-González, 2013, From Bayes to Tarantola: New insights to understand uncertainty in inverse prob- 
lems: Journal of Applied Geophysics, 98, 62-72.

Hansen, P. C., 1999, The l-curve and its use in the numerical treatment of inverse problems: IMM, Department of Mathematical Modelling, Technical Universityof Denmark.

Hansen, P. C., and D. P. O'Leary, 1993, The use of the l-curve in the regularization of discrete ill-posed problems: SIAM Journal on Scientific Computing, 14, 1487-1503.

Ijasan, O., C. Torres-Verdin, and W. E. Preeg, 2014, Inversion-based petrophysical interpretation of logging-while-drilling nuclear and resistivity measurements: Geophysics, 79, no. 3, D145-D159.

Key, K., 2012, Is the fast hankel transform faster than quadrature?: Geophysics, 77, no. 3, F21-F30.

Kong, J., 1972, Electromagnetic fields due to dipole antennas over stratified anisotropic media: Geophysics, 37, no. 6, 985-996.

Løseth, L., and B. Ursin, 2007, Electromagnetic fields in planarly layered anisotropic media: Geophysical Journal International, 170, no. 1, 44-80.

Merchant, G., R. Strickland, and C. Jackson, 1996, Enhanced resolution lwd resistivity logs using a new inversion technique: SPWLA 37th Annual Logging Symposium, 16-19.

Meyer, W. H., 1993, Inversion of the $2 \mathrm{mhz}$ propagation resistivity logs in dipping thin beds: Presented at the Trans SPWLA 34th Annual Logging Symposium, paper BB.

Nam, M. J., D. Pardo, and C. Torres-Verdín, 2008, Simulation of Dual-Laterolog Measurements in Dipping, Invaded and Anisotropic Formations Using a Fourier Series Expansion in a Non-Orthogonal System of Coordinates and a Self-Adaptive $h p$-Finite Element Method: Geophysics., 74, no. 1, E31-E43.

— 2010, Assessment of delaware and groningen effects on dual-laterolog measurements with a self-adaptive hp finite-element method: Geophysics, 75, no. 6, F143-F149. 
Newman, G. A., and D. L. Alumbaugh, 2002, Three-dimensional induction logging problems, part 2: A finite-difference solution: Geophysics, 67, no. 2, 484-491.

Pardo, D., M. Paszynski, C. Torres-Verdín, and L. Demkowicz, 2007, Simulation of 3D DC Borehole Resistivity Measurements with a Goal-Oriented hp Finite-Element Method. Part I: Laterolog and LWD: Journal of the Serbian Society for Computational Mechanics., 1, $62-73$.

Pardo, D., C. Torres-Verdín, M. J. Nam, M. Paszynski, and V. M. Calo, 2008a, Fourier series expansion in a non-orthogonal system of coordinates for simulation of 3D alternating current borehole resistivity measurements: Computer Methods in Applied Mechanics and Engineering, 197, 3836-3849.

Pardo, D., C. Torres-Verdín, and M. Paszynski, 2008b, Simulation of 3D DC borehole resistivity measurements with a goal-oriented $h p$ finite element method. Part II: Through casing resistivity instruments: Computational Geosciences, 12, no. 1, 83-89.

Streich, R., and M. Becken, 2011, Sensitivity of controlled-source electromagnetic fields in planarly layered media: Geophysical Journal International, 187, no. 2, 705-728.

Wait, J., 1966, Fields of a horizontal dipole over a stratified anisotropic half-space: Antennas and Propagation, IEEE Transactions on, 14, no. 6, 790-792.

Wait, J. R., 1951, The magnetic dipole over the horizontally stratified earth: Canadian Journal of Physics, 29, no. 6, 577-592.

Wang, T., and S. Fang, 2001, 3-D electromagnetic anisotropy modeling using finite differences: Geophysics, 66, no. 5, 1386-1398.

Wang, T., and J. Signorelli, 2004, Finite-difference modeling of electromagnetic tool response for logging while drilling: Geophysics, 69, no. 1, 152-160.

Zhang, J., R. L. Mackie, and T. R. Madden, 1995, 3-D resistivity forward modeling and 
inversion using conjugate gradients: Geophysics, 60, no. 5, 1312-1325.

Zhong, L., J. Li, A. Bhardwaj, L. C. Shen, and R. C. Liu, 2008, Computation of triaxial induction logging tools in layered anisotropic dipping formations: Geoscience and Remote Sensing, IEEE Transactions on, 46, no. 4, 1148-1163.

Zhou, J., 2012, LWD/MWD Resistivity Tool Parameters: http://www . maxwelldynamics. com/Pages/Resources.htm. 


\section{LIST OF FIGURES}

1 Original geometrical/geological setting and simplified model assumed for inversion. The well trajectory is a continuous $3 \mathrm{D}$ line penetrating a $1 \mathrm{D}$ subsurface model. . . . . . . . . . . . . . . . . . .

2 System of coordinates. . . . . . . . . . . . . . .

3 Relationship between the resistivity of the formation and the: (a) attenuation and (b) phase difference in a homogeneous formation for a particular frequency $(2 \mathrm{MHz})$ and transmitter-receiver spacing of a LWD resistivity device. . . . . . . . . . . . . . . . .

4 Synthetic example. Dip angle $=82^{\circ}$. Inversion results obtained for various logging instruments. Red lines identify actual resistivities, while blue lines correspond to estimated resistivities. . . . . . . . . . . .

$5 \quad$ Synthetic example. Dip angle $=82^{\circ}$. Error evolution as a function of iteration for various logging instruments. . . . . . . . . . . . . . . . . .

6 Synthetic example. Inversion results obtained with the first synthetic example for various dip angles. Red lines identify actual resistivities, while blue lines correspond to estimated resistivities. . . . . . . . . . . . . .

$7 \quad$ Synthetic example. Inversion results obtained for a TI formation. Red lines identify actual resistivities, while blue lines correspond to estimated resistivities. Dashed and continuous lines identify vertical and horizontal resistivities, respectively. . . . . . . . . . . . . . . . . .

8 Synthetic example with a thinly bedded formation (the thinnest layer has a thickness equal to $0.05 \mathrm{~m}$ ). Dip angle $=82^{\circ}$. Inversion results obtained for various logging instruments. Red and blue lines identify actual and estimated resistivities, respectively. . . . . . . . . . . . . . .

9 Synthetic example. Dip angle $=82^{\circ}$. Inversion results in terms of horizontal depth obtained in the presence of noise. Red lines identify actual resistivities, while blue lines correspond to inverted resistivities with their corresponding uncertainty bars. Apparent $(\log )$ resistivities for the shallowest and greatest depths of investigation are displayed in dark and light gray, respectively. . .

10 Synthetic examples with thinner layers (as thin as $0.05 \mathrm{~m}$ ). Dip angle $=82^{\circ}$. Inversion results obtained in the presence of noise. Red lines identify actual resistivities, while blue lines correspond to inverted resistivities with their corresponding uncertainty bars. Apparent (log) resistivities for the shallowest and greatest depths of investigation are displayed in dark and light gray, respectively. . . . . . . . . . . . . . . . .

11 Inversion results obtained from measurements acquired in field A with a conventional LWD tool in a highly deviated well. Apparent (log) resistivities for the shallowest and greatest depths of investigation are displayed in dark

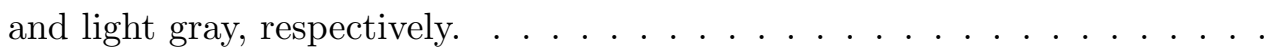


12 Inversion results of measurements acquired in field B with a conventional LWD tool in a highly deviated well. Apparent (log) resistivities for the shallowest and greatest depths of investigation are displayed in dark and light gray, respectively. . . . . . . . . . . . . . . . .

13 Relative data misfits (data residuals) of the resistivity logs. Top panel: field example A. Bottom panel: field example B. Left panel: data misfits corresponding to the initial solution, where the resistivity in each layer is selected as the average apparent resistivity. Right panel: data misfits obtained after executing the inversion algorithm. . . . . . . . . . . . 


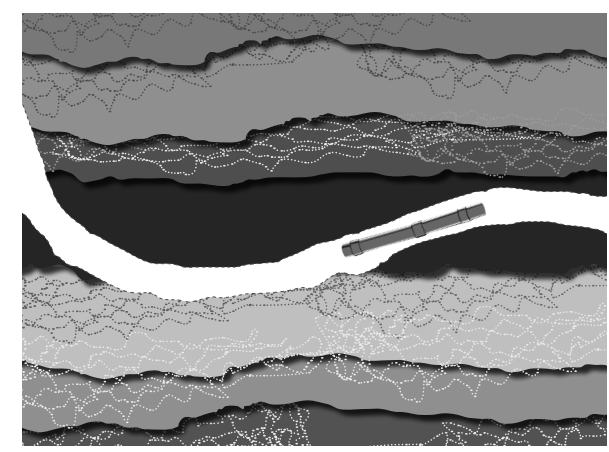

(a) Original Condition

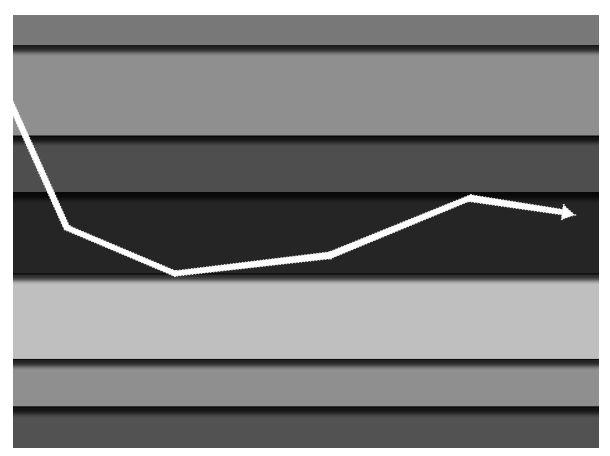

(b) Simplified Model

Figure 1: Original geometrical/geological setting and simplified model assumed for inversion. The well trajectory is a continuous 3D line penetrating a 1D subsurface model. 


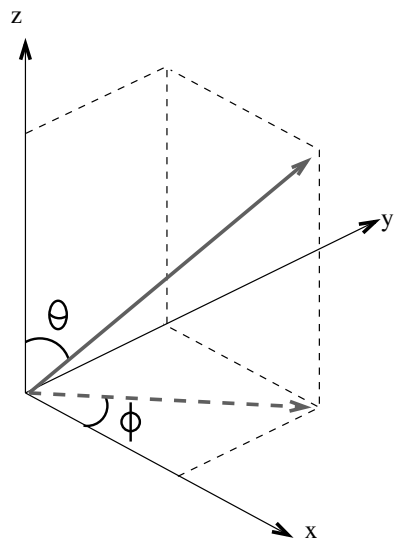

Figure 2: System of coordinates. 


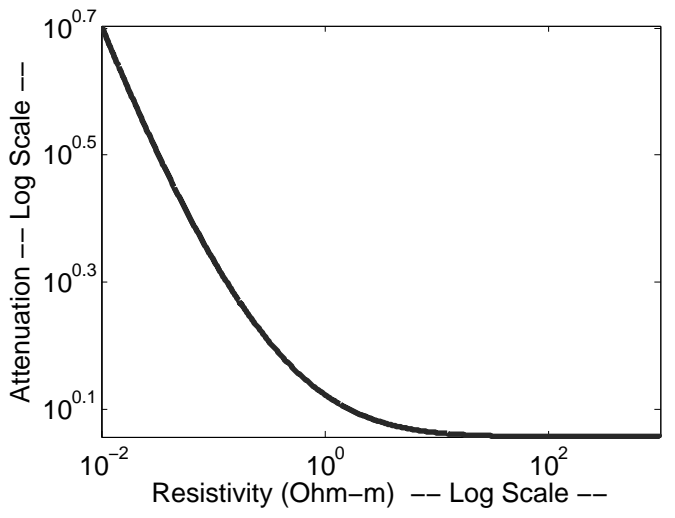

(a) Attenuation

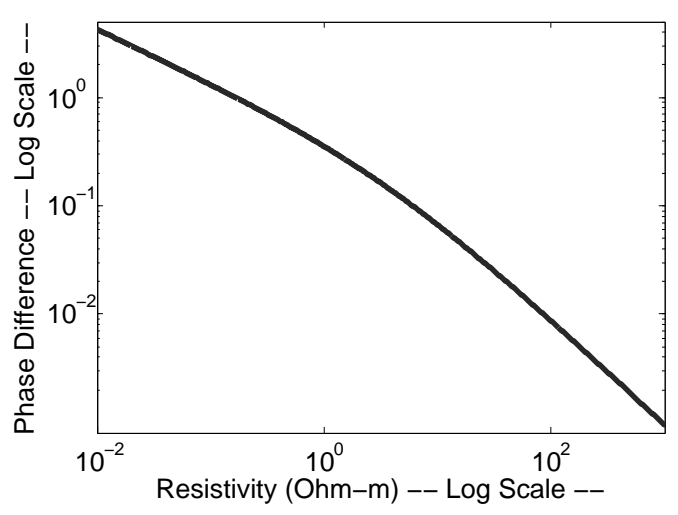

(b) Phase Difference

Figure 3: Relationship between the resistivity of the formation and the: (a) attenuation and (b) phase difference in a homogeneous formation for a particular frequency $(2 \mathrm{MHz})$ and transmitter-receiver spacing of a LWD resistivity device. 


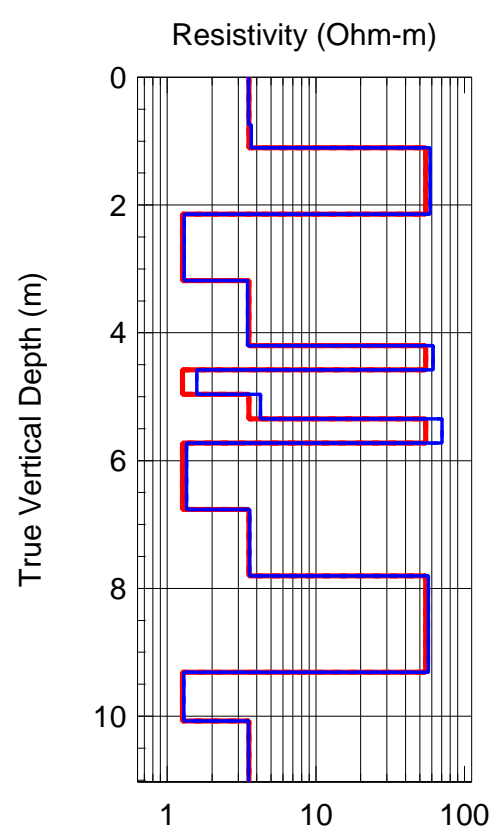

(a) Tool 1

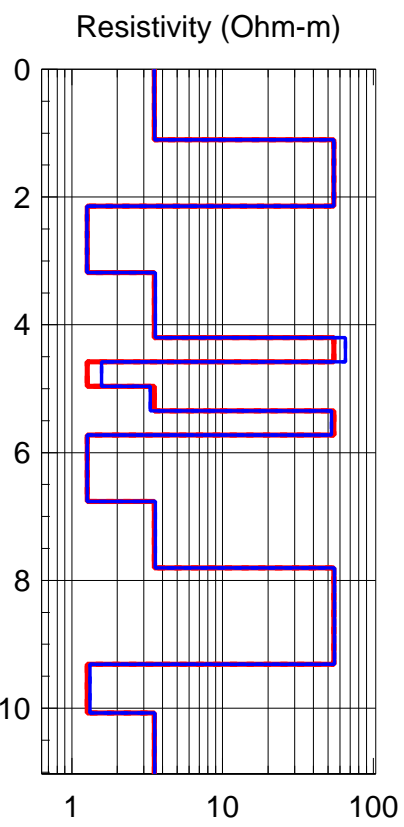

(b) Tool 3

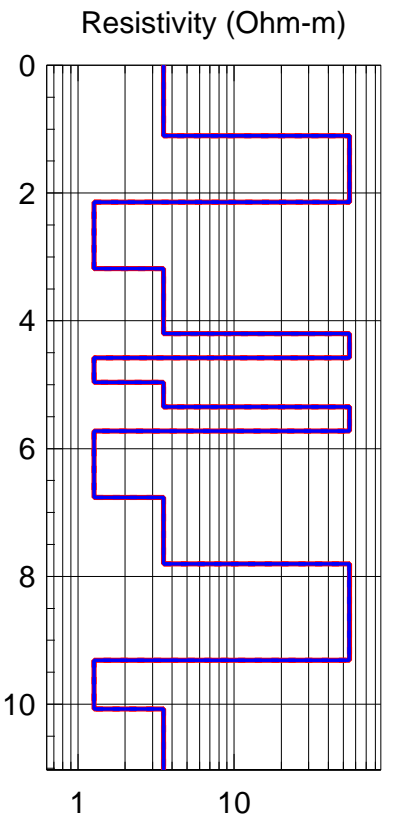

(c) Tool 4 (Tri-axial)

Figure 4: Synthetic example. Dip angle $=82^{\circ}$. Inversion results obtained for various logging instruments. Red lines identify actual resistivities, while blue lines correspond to estimated resistivities. 


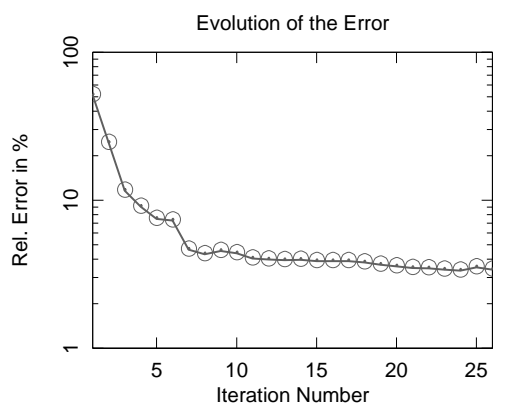

(a) Tool 1

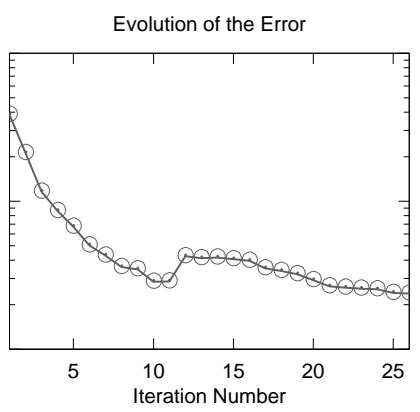

(b) Tool 3

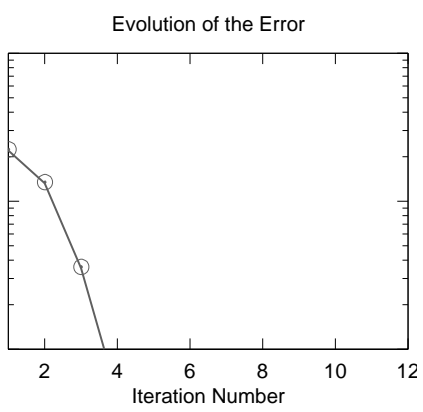

(c) Tool 4 (Tri-axial)

Figure 5: Synthetic example. Dip angle $=82^{\circ}$. Error evolution as a function of iteration for various logging instruments. 


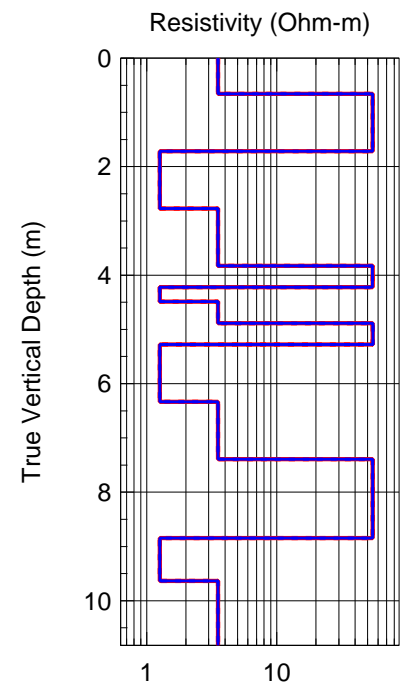

(a) $30^{\circ}$

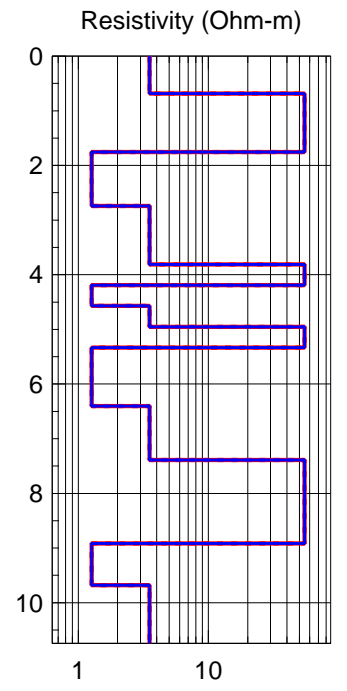

(b) $60^{\circ}$

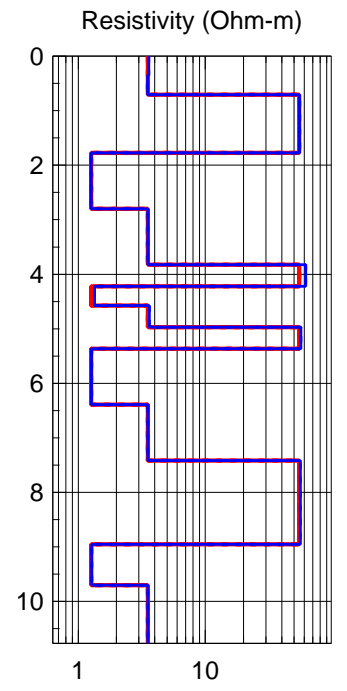

(c) $75^{\circ}$

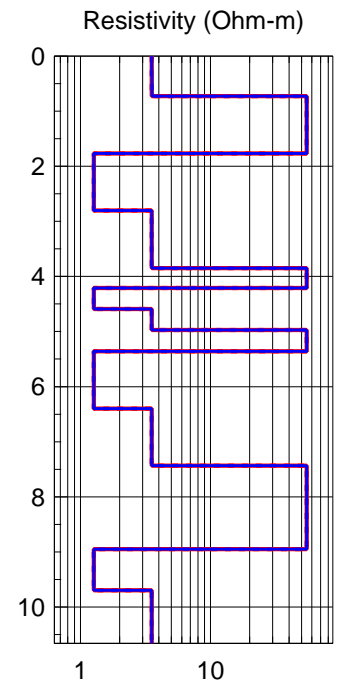

(d) $89^{\circ}$

Figure 6: Synthetic example. Inversion results obtained with the first synthetic example for various dip angles. Red lines identify actual resistivities, while blue lines correspond to estimated resistivities. 


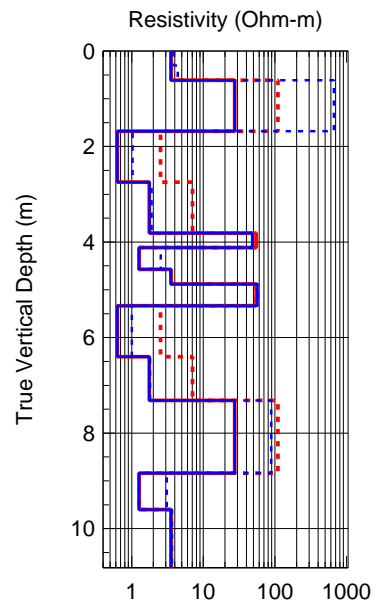

(a) Tool $3,0^{\circ}$

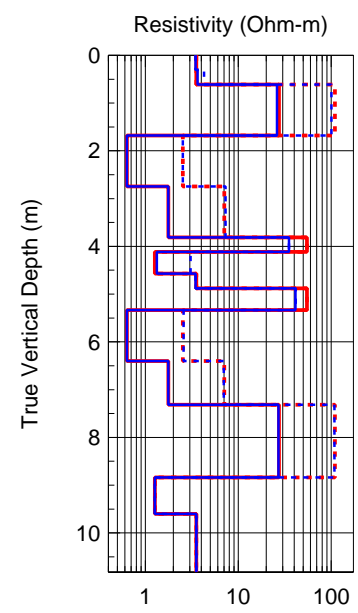

(d) Tool 4 (Tri-axial), $0^{\circ}$

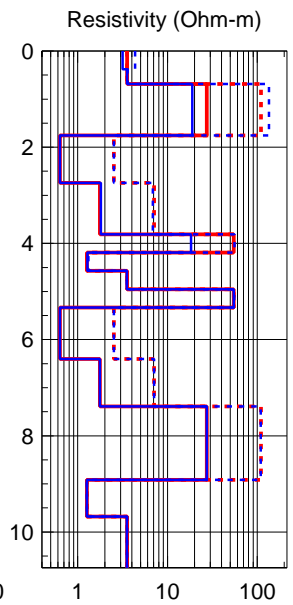

(b) Tool $3,45^{\circ}$

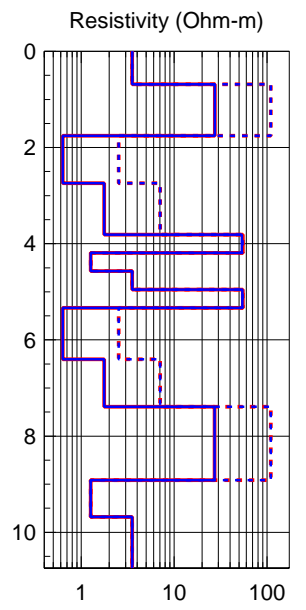

(e) Tool 4 (Tri-axial) $45^{\circ}($

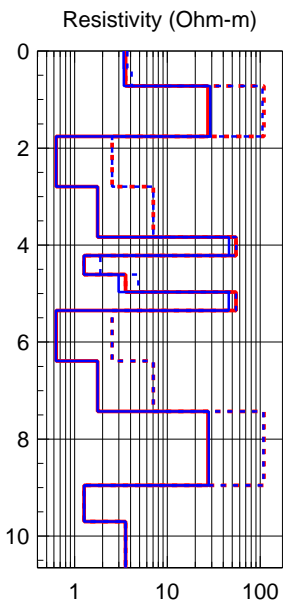

(c) Tool $3,82^{\circ}$

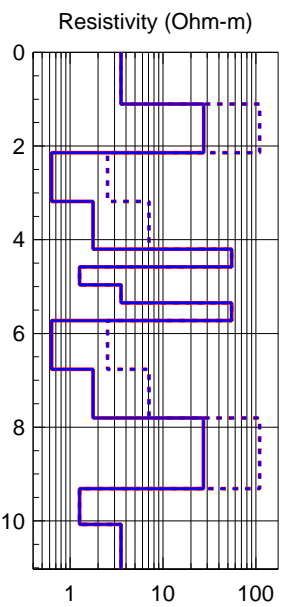

(f) Tool 4 (Tri-axial), $82^{\circ}$

Figure 7: Synthetic example. Inversion results obtained for a TI formation. Red lines identify actual resistivities, while blue lines correspond to estimated resistivities. Dashed and continuous lines identify vertical and horizontal resistivities, respectively. 


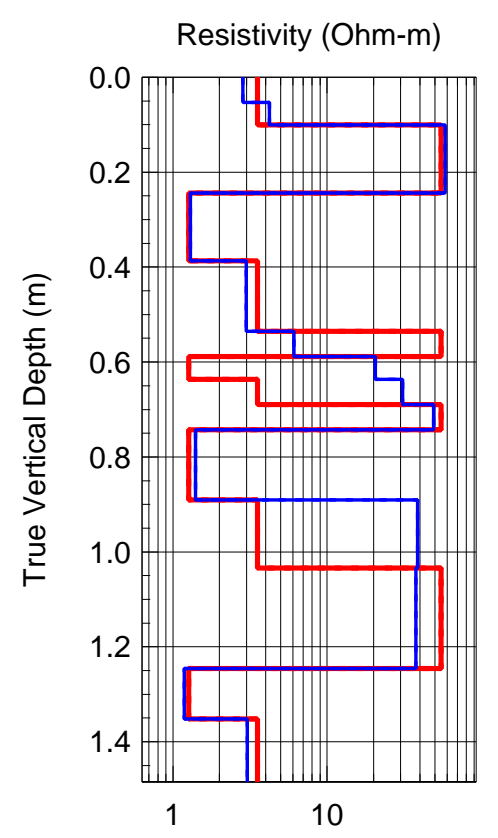

(a) Tool 1

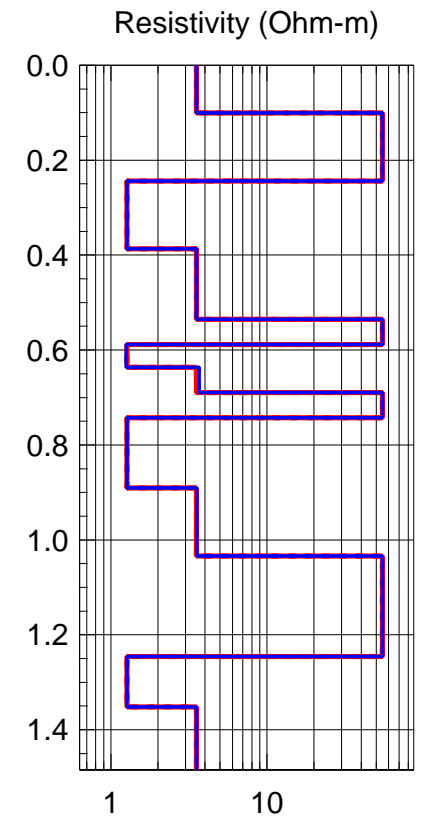

(b) Tool 3

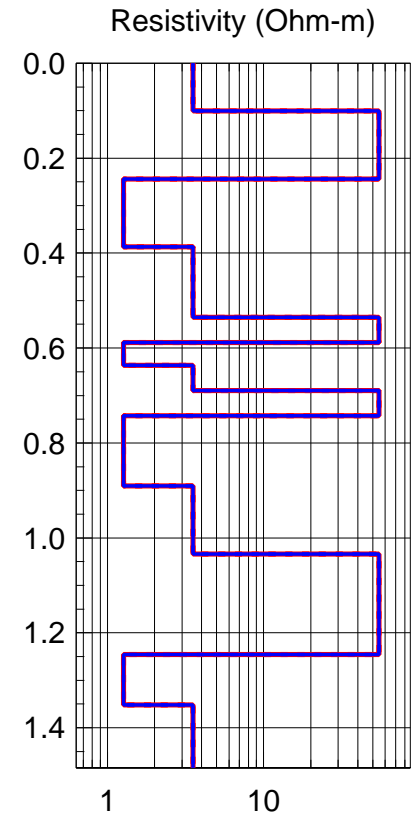

(c) Tool 4 (Tri-axial)

Figure 8: Synthetic example with a thinly bedded formation (the thinnest layer has a thickness equal to $0.05 \mathrm{~m}$ ). Dip angle $=82^{\circ}$. Inversion results obtained for various logging instruments. Red and blue lines identify actual and estimated resistivities, respectively. 


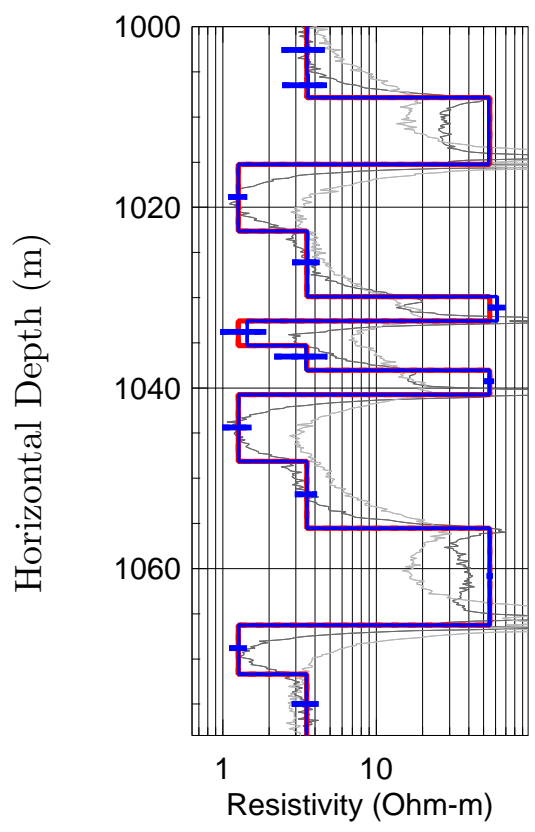

(a) $5 \%$ noise

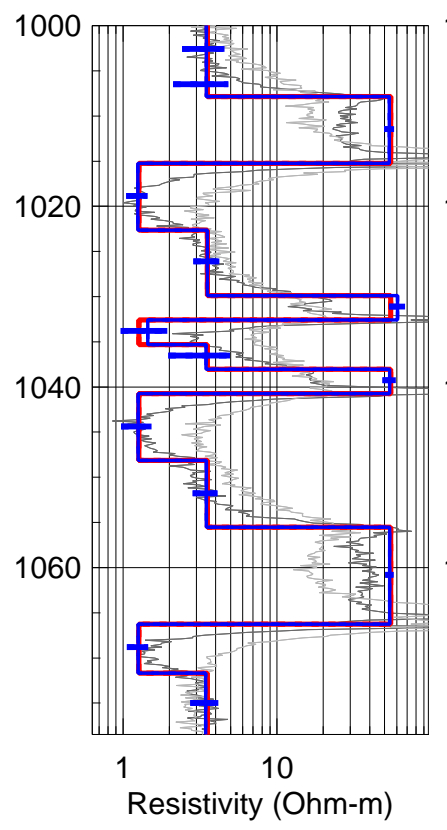

(b) $10 \%$ noise

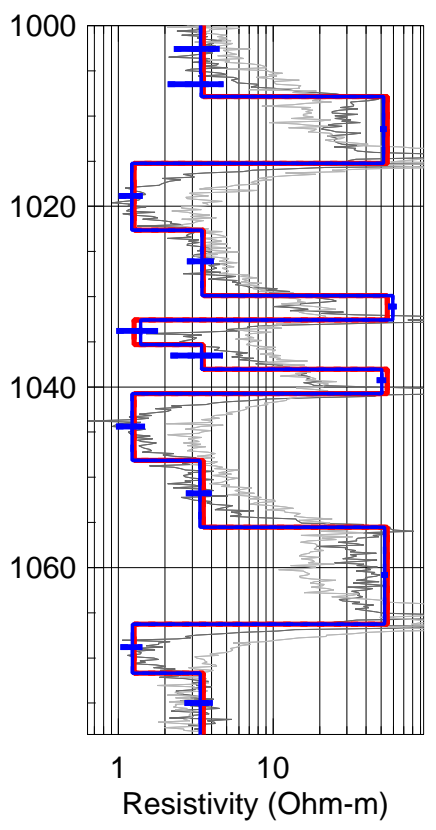

(c) $15 \%$ noise

Figure 9: Synthetic example. Dip angle $=82^{\circ}$. Inversion results in terms of horizontal depth obtained in the presence of noise. Red lines identify actual resistivities, while blue lines correspond to inverted resistivities with their corresponding uncertainty bars. Apparent (log) resistivities for the shallowest and greatest depths of investigation are displayed in dark and light gray, respectively. 


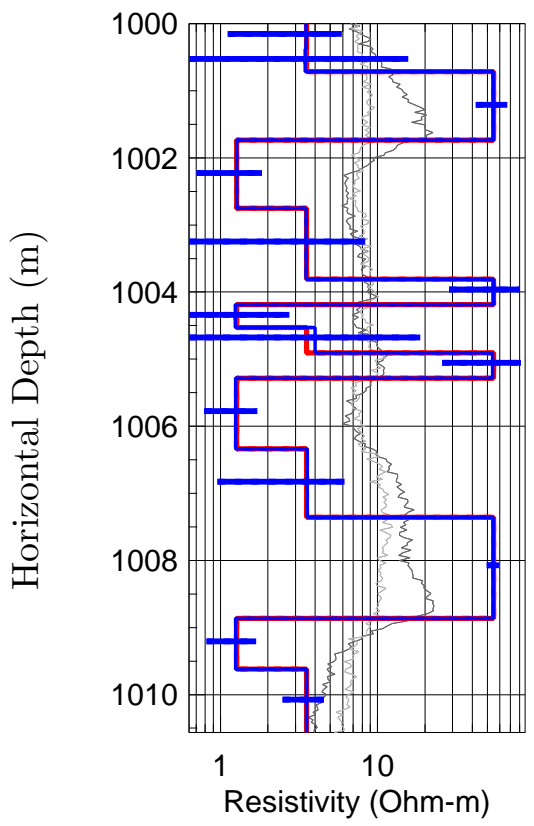

(a) $5 \%$ noise

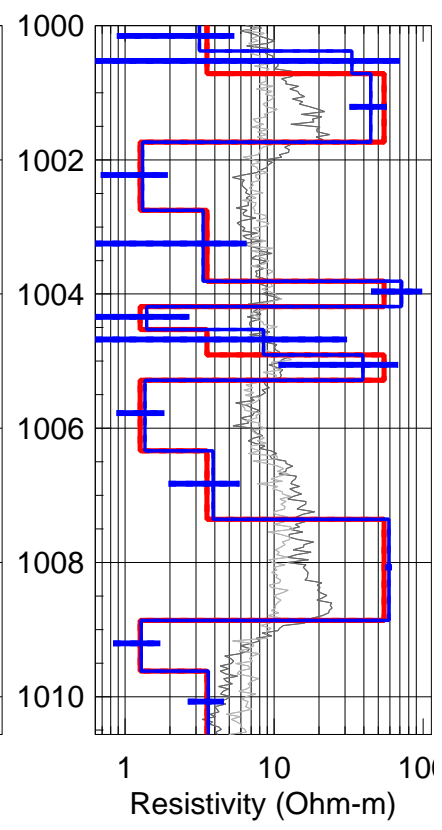

(b) $10 \%$ noise

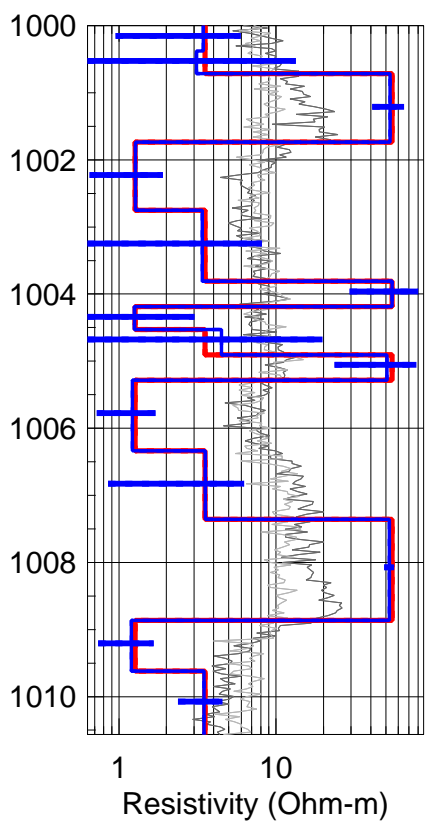

(c) $15 \%$ noise

Figure 10: Synthetic examples with thinner layers (as thin as $0.05 \mathrm{~m}$ ). Dip angle $=82^{\circ}$. Inversion results obtained in the presence of noise. Red lines identify actual resistivities, while blue lines correspond to inverted resistivities with their corresponding uncertainty bars. Apparent (log) resistivities for the shallowest and greatest depths of investigation are displayed in dark and light gray, respectively. 


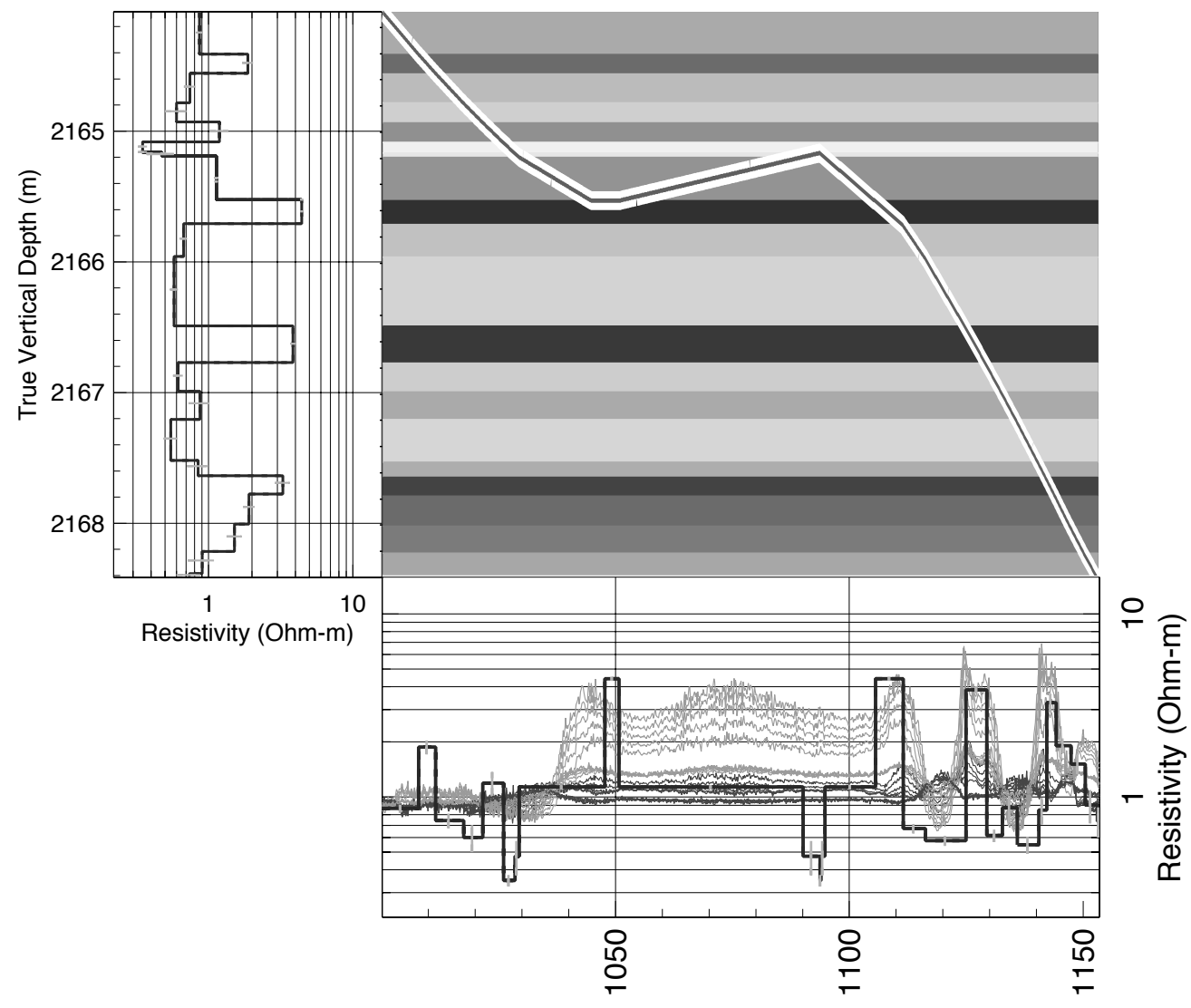

Figure 11: Inversion results obtained from measurements acquired in field A with a conventional LWD tool in a highly deviated well. Apparent (log) resistivities for the shallowest and greatest depths of investigation are displayed in dark and light gray, respectively. 


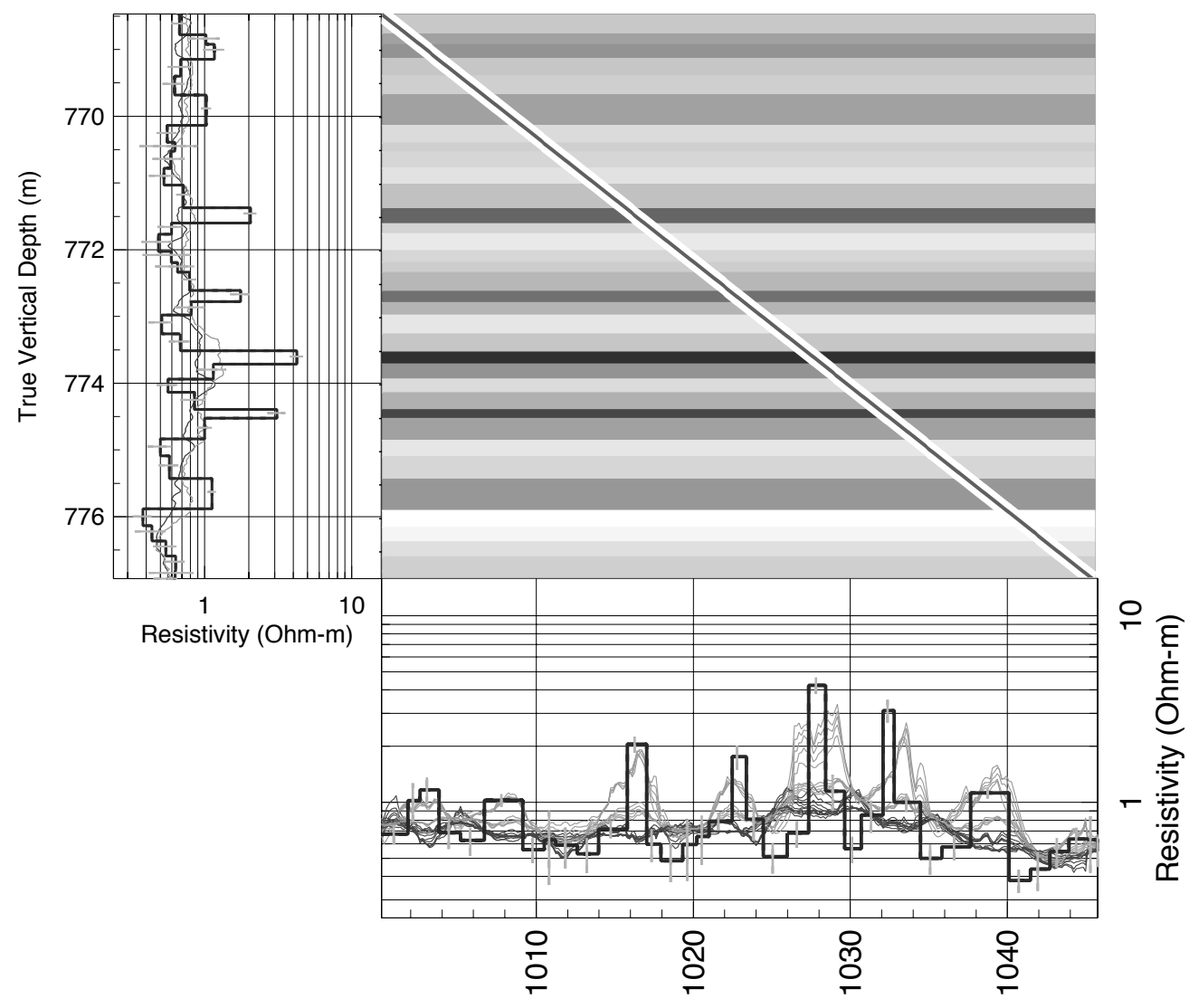

Figure 12: Inversion results of measurements acquired in field B with a conventional LWD tool in a highly deviated well. Apparent (log) resistivities for the shallowest and greatest depths of investigation are displayed in dark and light gray, respectively. 

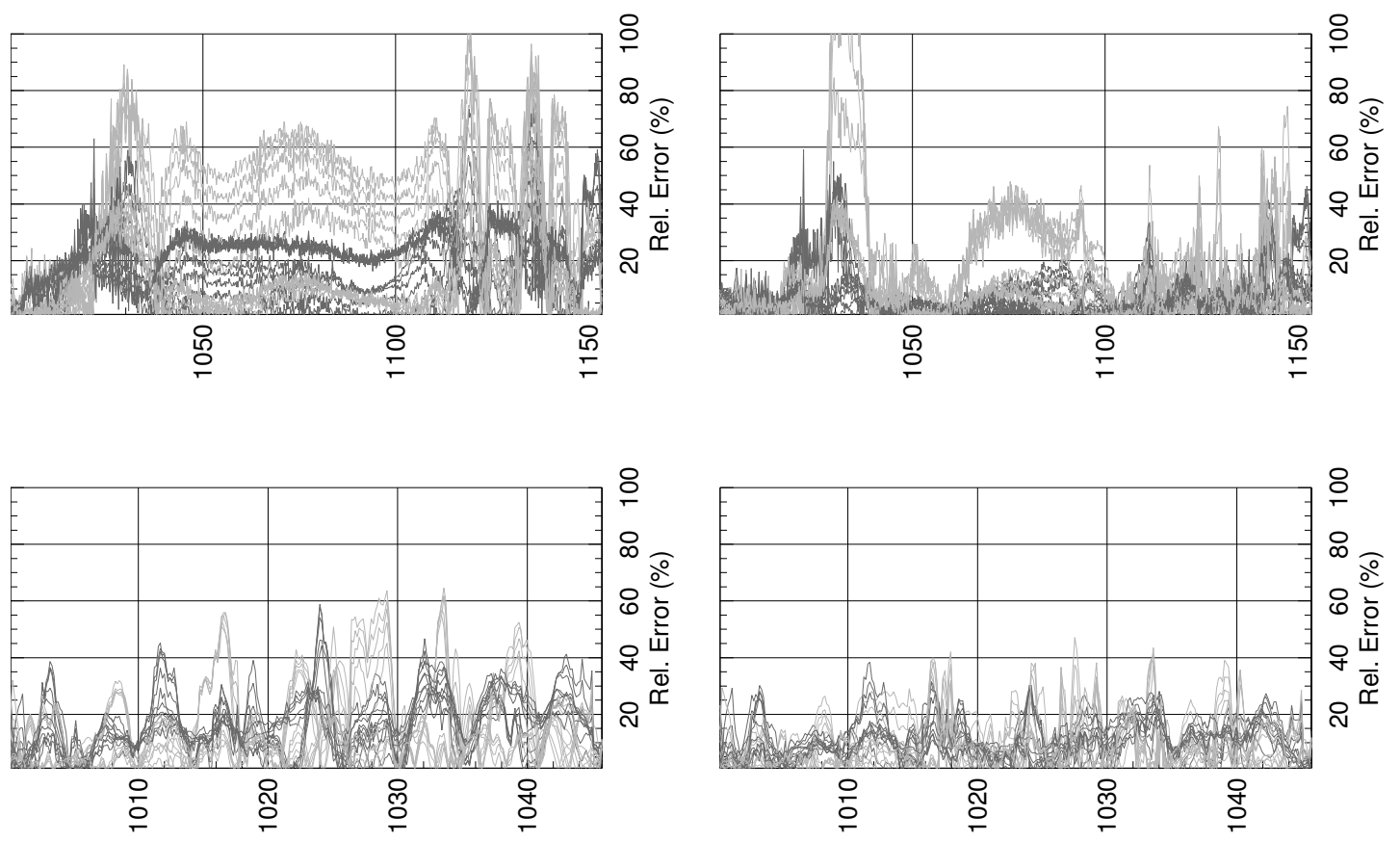

Figure 13: Relative data misfits (data residuals) of the resistivity logs. Top panel: field example A. Bottom panel: field example B. Left panel: data misfits corresponding to the initial solution, where the resistivity in each layer is selected as the average apparent resistivity. Right panel: data misfits obtained after executing the inversion algorithm. 


\section{LIST OF TABLES}

1 Specifications of the various commercial logging instruments considered in this paper. . . . . . . . . . . . . . . . . . . . . .

2 Computer time per iteration. Simulations were executed on a single core of a computer equipped with an Intel Core i7-3770 processor with 8 GB RAM. 


\begin{tabular}{|l|r|r|r|l|}
\hline $\begin{array}{l}\text { Logging } \\
\text { Instrument }\end{array}$ & Nr. TX & Nr. RX & Nr. Frequencies & Tool Type \\
\hline TOOL 1 & 4 & 2 & 2 & Conventional LWD \\
\hline TOOL 2 & 10 & 2 & 2 & Conventional LWD \\
\hline TOOL 3 & 10 & 2 & 3 & Conventional LWD \\
\hline TOOL 4 & 2 & 2 & 1 & Tri-axial \\
\hline
\end{tabular}

Table 1: Specifications of the various commercial logging instruments considered in this paper. 


\begin{tabular}{|l|r|r|r|r|r|r|r|}
\hline Example & $\begin{array}{r}\text { Nr. of } \\
\text { layers }\end{array}$ & $\begin{array}{r}\text { Nr. of } \\
\text { positions }\end{array}$ & $\begin{array}{r}\text { Nr. of } \\
\text { frequencies }\end{array}$ & $\begin{array}{r}\text { Nr. of } \\
\text { TX }\end{array}$ & $\begin{array}{r}\text { Nr. of } \\
\text { RX }\end{array}$ & $\begin{array}{r}\text { Time (s) } \\
\text { per iteration }\end{array}$ & $\begin{array}{r}\text { Time (ms) } \\
\text { per unit }\end{array}$ \\
\hline Figure 4a & 14 & 521 & 2 & 4 & 2 & 41.28 & 0.35 \\
\hline Figure 4b & 14 & 521 & 3 & 10 & 2 & 115.40 & 0.26 \\
\hline Figure 4c & 14 & 521 & 1 & 2 & 2 & 13.77 & 0.46 \\
\hline Figure 6a & 14 & 83 & 3 & 10 & 2 & 39.39 & 0.56 \\
\hline Figure 6d & 14 & 4014 & 3 & 10 & 2 & 1029.90 & 0.30 \\
\hline Figure 7c & 28 & 521 & 3 & 10 & 2 & 214.78 & 0.25 \\
\hline Figure 11 & 21 & 1021 & 2 & 10 & 2 & 392.95 & 0.46 \\
\hline Figure 12 & 36 & 301 & 2 & 10 & 2 & 167.50 & 0.39 \\
\hline
\end{tabular}

Table 2: Computer time per iteration. Simulations were executed on a single core of a computer equipped with an Intel Core i7-3770 processor with 8 GB RAM. 